\title{
Revisión de datos paleomagnéticos de flujos de lava asociados al Campo Volcánico Michoacán-Guanajuato: implicaciones sobre el comportamiento del campo magnético terrestre durante el Plio-Cuaternario
}

\author{
Ulises Díaz-Ortega, Rafael García-Ruiz*, Miguel Cervantes-Solano, \\ Avto Goguitchaichvili, Rafael Maciel-Peña, Juan Morales-Contreras y Rubén Cejudo-Ruiz
}

\author{
Laboratorio Interinstitucional de Magnetismo Natural, Instituto de Geofísica Unidad Michoacán, \\ Universidad Nacional Autónoma de México, Antigua Carretera a Pátzcuaro 8701, \\ Ex Hacienda de San José de la Huerta, 58190, Morelia, Mich., México. \\ *r.garcia@igeofisica.unam.mx
}

\section{RESUMEN}

La disponibilidad de edades radiométricas para el Campo Volcánico Michoacán-Guanajuato permite obtener un registro detallado de las variaciones del campo magnético de la Tierra durante los últimos $5 \mathrm{Ma}$. En el presente trabajo, analizamos 112 direcciones paleomagnéticas confiables respaldadas por igual número de edades radiométricas absolutas. Luego de descartar las polaridades geomagnéticas transicionales siguiendo el criterio de paleolatitudes menores a $45^{\circ}$, se calcularon las direcciones paleomagnéticas medias globales. En términos generales, se observa que la dirección media concuerda, dentro del intervalo de incertidumbres, con las direcciones paleomagnéticas esperadas obtenidas según los polos de referencia Plioceno-Cuaternario. La tasa de variación secular, estimada a través de la dispersión de los polos geomagnéticos virtuales calculados, y la anomalía de inclinación coinciden con los modelos de distribución latitudinal reportados para la latitud de $20^{\circ} \mathrm{N}$. Un hallazgo interesante, resultado de esta compilación de direcciones paleomagnéticas, es la posible evidencia de eventos geomagnéticos relativamente cortos o excursiones dentro de los Crones de Matuyama y Brunhes, los cuales pueden ayudar a refinar la escala de tiempo de inestabilidad geomagnética. Además, se presenta una compilación inédita y una descripción minuciosa de más de 1000 aparatos volcánicos realizada por el Dr. Víctor Hugo Garduño Monroy que, sin duda alguna, servirá como una guía para los estudios paleomagnéticos durante las siguientes décadas.

Palabras clave: Campo Volcánico Michoacán-Guanajuato; paleomagnetismo; variación paleosecular; eventos geomagnéticos cortos; México.

\section{ABSTRACT}

The great availability of radiometric ages for the MichoacanGuanajuato Volcanic Field allows obtaining a detailed record of the Earth's magnetic field variations during the last $5 \mathrm{Ma}$. In this work, we analyze 112 reliable paleomagnetic directions supported by the same number of absolute ages. After discarding the transitional geomagnetic polarities, following the criterion of paleolatitudes less than $45^{\circ}$, the global mean paleomagnetic directions were calculated. In general terms, it is observed that the mean direction agrees, within the uncertainty interval, with the expected paleomagnetic directions obtained according to the Pliocene-Quaternary reference poles. The secular variation rate, estimated through the dispersion of the calculated virtual geomagnetic poles, and the inclination anomaly coincide with the latitudinal distribution models reported for the latitude of $20^{\circ} \mathrm{N}$. An interesting finding, resulting from this compilation of paleomagnetic directions, is the possible evidence of relatively short geomagnetic events or excursions within the Matuyama and Brunhes chrones, which may help to refine the geomagnetic instability time scale. Moreover, an unpublished compilation and a detailed description of more than 1000 volcanic buildings by Dr. Victor Hugo Garduño Monroy are presented, which will undoubtedly serve as an authentic guide for paleomagnetic studies during the following decades.

Key words: Michoacán-Guanajuato Volcanic Field; paleomagnetism; paleosecular variation; short geomagnetic events; Mexico.

\section{INTRODUCCIÓN}

Conocer la variación del campo geomagnético en el tiempo es esencial para entender las condiciones del núcleo líquido de la Tierra y la frontera núcleo-manto. Los registros paleomagnéticos y arqueomagnéticos permiten estimar de manera indirecta las condiciones de las profundidades de la Tierra en diferentes escalas de tiempo, en particular, los cambios en las condiciones en el manto inferior pueden ser los responsables del comportamiento geomagnético a largo plazo y de la frecuencia en la ocurrencia de las inversiones geomagnéticas (Gubbins, 1988). Por otro lado, los estudios de variación paleosecular (PSV, paleosecular variation por sus siglas en inglés) y campo promediado en tiempo (time averaged field TAF) están siendo considerados como factores decisivos para comprender el comportamiento del campo magnético de la Tierra. El TAF evidencia los desvíos del

Díaz-Ortega, U., García-Ruiz, R., Cervantes-Solano, M., Goguitchaichvili, A., Maciel-Peña. R., Morales-Contreras, J., Cejudo-Ruiz, R., 2021, Revisión de datos paleomagnéticos de flujos de lava asociados al Campo Volcánico Michoacán-Guanajuato: implicaciones sobre el comportamiento del campo magnético terrestre durante el Plio-Cuaternario: Revista Mexicana de Ciencias Geológicas, v. 38, núm. 3, p. 259-271. 
dipolo geocéntrico axial (GAD, por sus siglas en inglés), el modelo más básico del campo enteramente paleomagnético (Lawrence et al., 2009). La iniciativa del TAF comenzó con crear una base de datos de observaciones geomagnéticas para los últimos $5 \mathrm{Ma}$ (Tauxe y Kent 2004, Lawrence et al., 2006; Johnson et al., 2008).

Johnson et al. (2008) publicaron una síntesis de una nueva generación de datos paleomagneticos, reportando la dispersión del polo virtual geomagnético versus latitud. También se reportaron dos ejemplos de modelos de dispersión de VGP (Virtual Geomagnetic Poles, por sus siglas en inglés) con respecto a la latitud; el Modelo G y el TK03. Es de llamar la atención que la dependencia latitudinal de la dispersión para el conjunto de datos compilados por Johnson et al. (2008) parecía ser poco significativa. Los datos provenientes de bajas latitudes parecen estar más dispersos, mientras que los que provienen de latitudes altas están menos dispersos. Sin embargo, la contribución de Lawrence et al. (2009) sugiere que el parámetro de la dispersión $S_{B}$ no depende de la latitud, y subraya la importancia de incluir nuevos conjuntos de datos de buena calidad. Es de resaltar que la visión de la dependencia de la latitud con la dispersión de VGP presentadas por Johnson et al. (2008) depende fundamentalmente de un conjunto de datos cercanos a los $20^{\circ} \mathrm{N}$, incluidos una gran cantidad de datos del centro y oeste de México.

La Faja Volcánica Transmexicana (FVTM) es una zona con una orientación E-O localizada entre las latitudes $19^{\circ}$ y $20^{\circ} \mathrm{N}$, que se extiende aproximadamente $1000 \mathrm{~km}$ desde el Pacifico hasta el Golfo de México. Su origen está relacionado con la subducción de la placa de Cocos por debajo de la norteamericana (Ferrari et al., 2012). Algunos de sus grandes estratovolcanes como el Popocatépetl y Colima, son reconocidos por su gran tamaño y actividad volcánica reciente. La mayoría de los volcanes que comprenden la FVTM son conos de escoria monogenéticos, y aunque la total cantidad total no se conoce con exactitud, se estima que son mas de 3000 (Hasenaka y Carmichael, 1985b).

Probablemente no existe otro arco magmático NeógenoCuaternario de subducción en el mundo que comprenda tal cantidad de conos de escoria y se puede decir que, en este sentido, la FVTM es única. Existen conos de escoria en la FVTM, estos no se distribuyen de manera uniforme. Dos áreas muestran las mayores concentraciones de volcanes monogenéticos: La Sierra Chichinautzin, al sur de la Ciudad de México y el Campo Volcánico Michoacán-Guanajuato (CVMG). Este último contiene el único registro de erupciones históricas, el Volcán Jorullo en 1759, y el Volcán Paricutín en 1943. Individualmente, los volcanes monogenéticos que forman el CVMG tienen pequeños volúmenes, pero en su conjunto alcanzan un área extensa.

El presente trabajo reporta una base de datos del campo geomagnético promediado en tiempo y de variación paleosecular basada en estudios paleomagneticos y magnetismo de rocas realizados en 112 flujos de lava asociados al CVMG.

\section{Campo Volcánico Michoacán-Guanajuato}

La Faja Volcánica Trans-Mexicana (FVTM) es un extenso arco volcánico localizado en la zona central del territorio mexicano, el cuál es uno de los arcos volcánicos más variados y con suma complejidad dentro de la región del Circum-Pacifico (Kennett el al., 1977, Ferrari, 2000), delimitado en el occidente por el rift de Colima, sobrepuesto al norte con el Bloque Jalisco y convive al oeste con la Sierra Madre Occidental (SMO) e incluye grandes y variados volcanes compuestos, pequeños conos monogenéticos y centros volcánicos silícicos, con variedades de rocas alcalinas emplazadas sobre toda la FVTM (Tabla 1). Tiene una longitud aproximada de $1000 \mathrm{~km}$, se localiza entre las latitudes $18^{\circ} 30^{\prime} \mathrm{N}$ y $21^{\circ} 30^{\prime} \mathrm{N}$, cubre una extensión de 160000 km², con un espesor variable entte los 90 y $230 \mathrm{~km}$ (Ferrari 2000; Ferrari et al., 2012). De acuerdo por el tipo de vulcanismo y a su composición química, este complejo volcánico se divide en la zona occidental, central y oriental. El origen del vulcanismo en la región se remonta a un periodo previo al Mioceno y se debe al proceso de subducción de la placa de Farallón al oeste-suroeste de la placa norteamericana. Dicho proceso continua hasta el presente con la subducción de la placa de Cocos y la placa de Rivera, ambas remanentes de la placa de Farallón (Atwater, 1970; Mammerickx y Klitgord, 1982, Clark et al., 1982).

El Campo Volcánico Michoacán-Guanajuato (CVMG) localizado en la parte central este de la FVTM (Figuras 1 y 2 ), abarcando un área de $40000 \mathrm{~km}^{2}$, se encuentra comprendido entre las latitudes $19^{\circ}$ y $20.75^{\circ} \mathrm{N}$ y las longitudes $102.666^{\circ}$ y $100.333^{\circ} \mathrm{E}$ (Hasenaka- Carmichael, 1985a). Está conformado por más de 1000 aparatos volcánicos, de los cuales cerca de 900 son conos de lava o ceniza con una distribución espacial aleatoria y sin mostrar una orientación preferente, se identifican 43 domos, 22 maars o anillos de toba, 13 volcanes jóvenes con conos en la cima y 61 flujos de lava.(Hasenaka-Carmichael, 1985b). Además de los centros volcánicos pequeños existen en la zona unos 300 volcanes de tamaño medio, que son mayormente volcanes tipo escudo cuyos radios rondan los $10 \mathrm{~km}$, estos son responsables de las mayores emisiones de lava en la región (Ban et al., 1992). La región norte del CVMG está caracterizada por la presencia de una gran cantidad de volcanes medianos, los cuales, a partir de su geomorfología, se puede inferir que son más antiguos que su contraparte en la región sur (Hasenaka y Carmichael, 1985a). Los datos radiométricos muestran también una tendencia de migración de la actividad volcánica en la zona central hacia $100 \mathrm{~km}$ al sur durante el periodo entre 1 y $2 \mathrm{Ma}$, dicha migración no fue gradual si no abrupta lo que implica que en la región existen dos grupos de volcanes con diferentes edades (Ban et al., 1992). La mayor concentración de conos se localiza a $250 \mathrm{~km}$ de la TMA (Trinchera Media Americana) e incluye el cono de escoria más joven de la región, el volcán Paricutín en la zona noroeste. Aproximadamente el $75 \%$ de los conos de escoria se localizan entre los 200 y $300 \mathrm{~km}$ de la TMA, más allá de los $250 \mathrm{~km}$ la frecuencia de los eventos decrece a excepción de una pequeña aglomeración a los $380 \mathrm{~km}$. El Valle de Santiago es la única zona en la que se presentaron eventos de conos de actividad explosiva y maars. Asumiendo una distribución homogénea en todo el campo volcánico, se puede calcular la densidad de eventos, resultando en 2.5 aparatos $/ 100 \mathrm{~km}^{2}$, mientras que la mayor densidad a nivel local se localiza en la zona del Paricutín que alcanza 11 aparatos / $100 \mathrm{~km}^{2}$ (Hasenaka y Carmichael, 1985b). Las lavas con edades menores a 40000 años presentan una composición calco-alcalina con apenas una mayor concentración de silicio en comparación con aquellas lavas de edades superiores que son mayormente alcalinas (Hasenaka y Carmichael, 1987).

\section{ESTUDIOS PALEOMAGNÉTICOS REALIZADOS EN EL CVMG}

Los primeros estudios paleomagnéticos realizados en regiones que pertenecen al CVMG fueron algunas investigaciones que incluyeron sitios de estudio que actualmente pueden considerarse como pertenecientes al CVMG. Sin embargo, estos sitios no fueron estudiados por su pertenencia a él, sino más bien como parte de la FVTM para investigar la variación paleosecular del CMT en distintos periodos, por ejemplo; Böhnel y Negendank (1981), Alva-Valdivia et al. (1991). Es muy notable que a partir de la publicación de los trabajos de Hasenaka y Carmichael (1985a, 1985b) en donde describen más de mil centros volcánicos y establecen correlaciones geocronológicas, comienzan a realizarse investigaciones paleomagnéticas en sitios específicos del CVMG. En los siguientes párrafos se enlistan en orden cronológico y se describe de forma breve los trabajos previos realizados con un enfoque paleomagnético en sitios de esta región. 


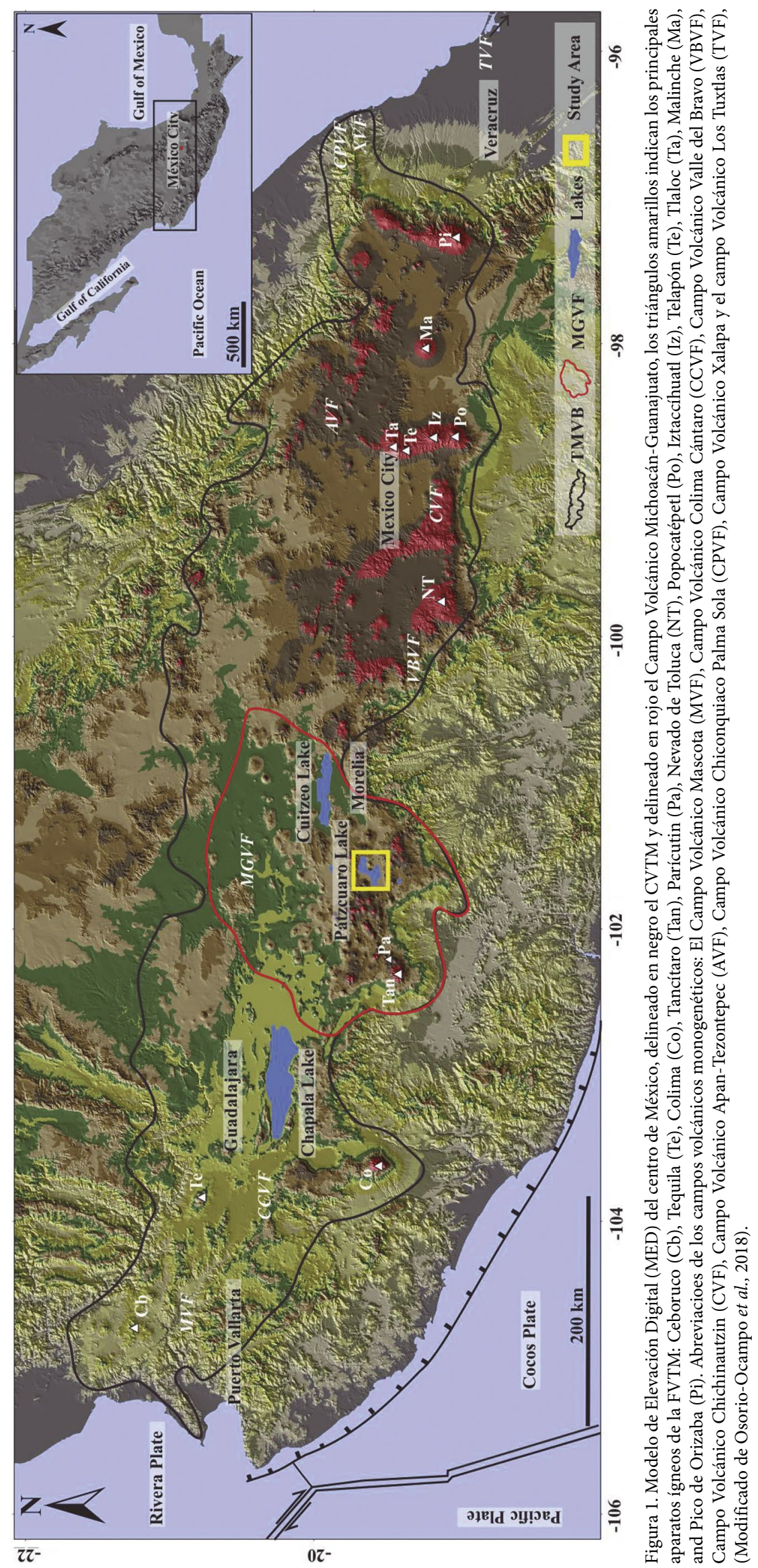




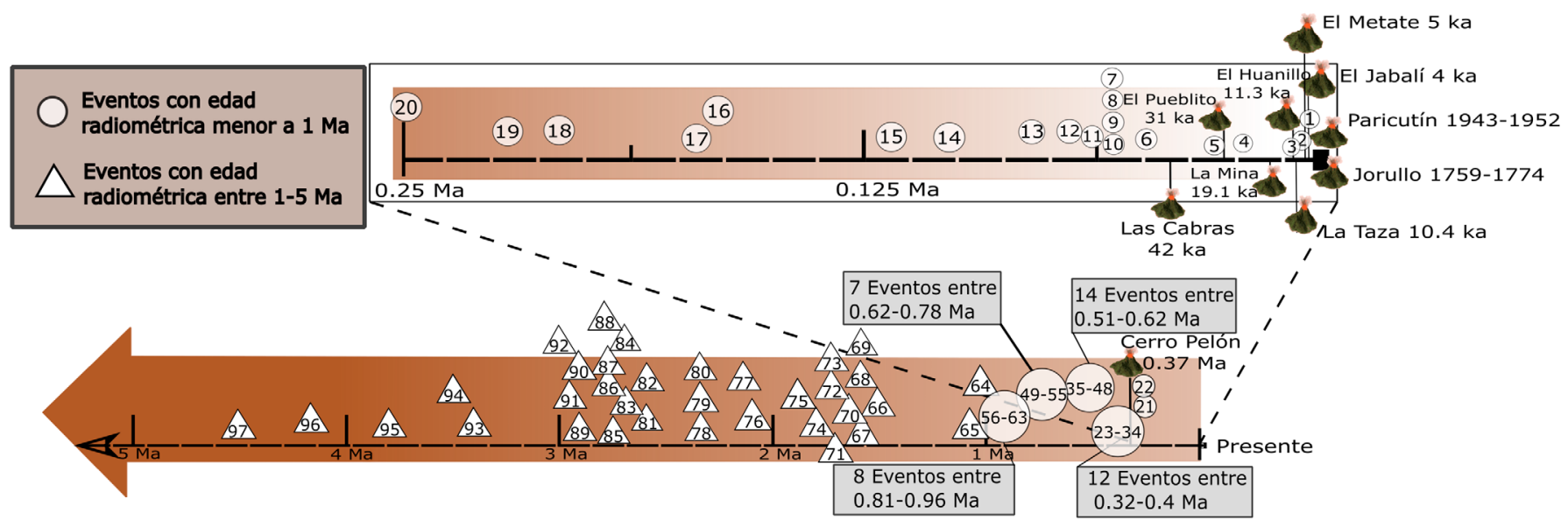

Figura 2. Distribución de edades radiométricas disponibles a lo largo del Campo Volcánico Michoacán-Guanajuato. 1. Tetimpa, 2. AG2, 3. Tres Cruces, 4. El Estribo, 5. JO-12 (El Guayabo), 6. Tac-17 (C. Las Animas), 7. Cerro Paracho, 8. Tac-22 (Alberca), 9. Tac-23 (Alberca 2), 10. Tac-02 (C. El Sosal), 12. EG, 13. TAN08, 14. Tan10, 15. TAN02, 16. AL2, 17. Tan11, 18. El Picacho, 19. Tan2, 20. Tac-18 (C. Chepelopez), 21. Tan6, 22. CD, 23. AH, 24. Tac-09 (C. Flogopitario), 25. CF, 26. TCP-06, 27. TAN01, 28. BJ, 29. Tan7, 30. TAN07, 31. Tac-03B (C. Nombre de Dios), 32. TCP-07, 33. Tac-03A (C. Nombre de Dios), 34. TCP-05, 35. Tan3, 36. TCP-03, 37. Tac-25 (C. Chocolate), 38. TCP-10, 39. Tac-14 (C. La Barra), 40. Yahuarato, 41. Buenavista Tomatlan, 42. M-01, 43. M-07, 43. Tac-05 (C. Zihuatanejo), 44. TCP- 08, 45. Tac-10 (C. Cantaro), 46. Tan9, 47. Tan8, 48. TCP-02, 49. Tac-24 (C. Partido), 50. AZ, 51. Tac-15 (C. Las Flores), 52. M-09, 53. M-10, 54. TAN06, 55. TCP-01, 56. JO-07 (Mata de Plátano), 57. Cerro Sanambo, 58. TAN03, 59. JO-15 (C. El Pelón), 60. TAN05, 61. CI, 62. TCP-11, 63. Tac-13 (C. El Jabali), 64. JO-17 (Mesa El Palmar), 65. CN, 66. qcnCOP, 67. 6-Cerro Grande La Piedad, 68. qcrCG, 69. qcnSM, 70. Tac-08 (C. La Ventana), 71. Tac-07 (C. Petembo), 72. Tac-12 (Mesa El Encinal), 73. Tac-11 (M. Calzada), 74. Tac-20 (C. El Salitrillo), 75. 9-Brinco del Diablo, 76. 3-Cerro Culiacán, 77. pcnBDI, 78. M-04, 79. M-05, 80. M-06, 81. M-08, 82. pcnCAL, 83. Tac-26 (C. San Jose), 84. Tac-21 (C. Hueco), 85. JO-19 (El Cangrejo), 86. M-02, 87. M-03, 88. pcrSOL, 89. JO-16 (Mesa Serrato), 90. pcrHUA, 91. JO-13 (Mesa Vicente), 92. pcnPAJ, 93. pcnPSA, 94. BL, 95. pcnGUA, 96. Tac-19 (C. La Cruz 2), 97. pcnVIR, 98. JO-14 (aplitic dike), 99. JO-18 (Pluton La Huacana).

Rosas-Elguera y Urrutia-Fucugauchi (1992) realizarón un estudio paleomagético y radiométrico en 14 sitios volcánicos localizados en la zona geotérmica de Ixtlán de los Hervores-Los Negritos en Michoacán. Obtuvieron direcciones y polaridades que les permitieron, con ayuda de edades radiométricas ya conocidas y cinco nuevos fechamientos, establecer una correlación estratigráfica, concluyendo que la actividad volcánica en esa zona geotérmica se desarrolló durante un intervalo que comprende desde el Mioceno Tardío hasta el Cuaternario. Reportaron dos registros de los eventos de polaridad geomagnética Reunión (2.01-2.04 Ma y 2.12-2.14 Ma).

Delgado-Granados et al. (1995) con el fin de establecer correlaciones estratigráficas y establecer la temporalidad de la actividad volcánica, realizaron un estudio paleomagnético y radiométrico analizando muestras de rocas volcánicas de 32 sitios pertenecientes al CVMG en el sector oeste de la FVMT. Con las edades radiométricas y las polaridades magnéticas obtenidas, establecieron que la actividad volcánica tuvo lugar del Mioceno al Pleistoceno sin evidencia de movimientos tectónicos significativos desde esa época.

González et al. (1997) publican resultados de un estudio de variación secular y de paleointensidad del CMT durante de los últimos 30000 años realizado en 13 flujos de lava distintos con edades radiométricas conocidas, distribuidos en la región central de México. Seis de esos flujos pertenecen al CVMG. Siguiendo las metodologías Thellier y Thellier (1959) y la de Shaw (1974) obtuvieron 13 nuevas determinaciones de paleointensidades y encuentran también que la variación secular observada en los últimos 3000 años tiene una amplitud relativamente alta.

Cifuentes y Urrutia-Fucugauchi (1999) presentan los resultados de un estudio paleomagnético realizado en 13 diferentes unidades volcánicas localizadas en la región de Valle de Santiago del CVMG. A partir de las polaridades encontradas y de la edad radiométrica disponible asociada al vulcanismo de la región, concluyen que la actividad volcánica que dio origen a las unidades estudiadas se desarrolló predominantemente durante el Cron de Brunhes $(<0.78 \mathrm{Ma})$. Reportan también que la posición promedio del polo obtenida tiene una diferencia angular con respecto a la dirección esperada y que podría ser el resultado de la deformación regional en este sector de la TMVB.

Rosas-Elguera et al. (2003) llevan a cabo un estudio paleomagnético y radiométrico en 12 sitios localizados en el bloque Michoacán, en la parte occidental del CVMG para establecer mediante las posiciones del polo geomagnético la existencia de movimientos tectónicos regionales. Obtienen edades entre 31.6 a $8.39 \mathrm{Ma}$, y a partir de los datos paleomagnéticos encuentran evidencia de una posible rotación vertical cercana a los $24^{\circ}$ del bloque Michoacán.

Este mismo año Morales et al. (2003) realizaron un estudio piloto de paleointensidad en los flujos históricos del volcán Paricutín de 1943 y 1948 utilizando el método de microondas desarrollado por Kono y Ueno (1977). Sus resultados arrojan valores significativamente distintos a los esperados por lo que, dado lo reciente de las erupciones, advierten que esta diferencia puede deberse al método utilizado para la determinación de la paleointensidad y sugieren de utilizar factores de corrección como el de ritmo de enfriamiento para muestras naturales.

Urrutia-Fucugauchi et al. (2004) publicaron un estudio paleomagnético y de magnetismo en rocas provenientes de flujos de lava del volcán Paricutín. Se trata de 12 sitios que provienen de siete eventos eruptivos ocurridos entre 1943 y 1952. Sus resultados indican que, al tratarse de lavas muy jóvenes, la magnetización presente en ellas es de origen termal primario. Encuentran también que las inclinaciones magnéticas promedio por sitio son más bajas que lo que se espera según el registro del Observatorio Geomagnético de Teoloyucan y el modelo geomagnético de referencia. Atribuyen estas anomalías a una posible deformación interna del flujo de lava durante al emplazamiento.

Posteriormente Goguitchaichvili et al. (2005) reportan un estudio de paleointesidad realizado también en lavas del volcán Paricutín emplazadas durante el período entre 1943 y 1948. Las paleointensidades se obtuvieron mediante el método de microondas de Kono y Ueno (1977) 
y variaciones del método de Thellier y Thellier (1959). Sus resultados, mostraron una dispersión alta y con valores de PI significativamente diferentes del valor esperado. Concluyen que la discrepancia entre sus resultados obtenidos y los valores esperados puede deberse al hecho de que la magnetización de las muestras estudiadas no es puramente de origen termomagnética. Cuestionan también la reproducibilidad de los métodos debido a la falta de criterios objetivos que permitan discriminar las determinaciones confiables.

En el trabjao publicado por Conte-Fasano et al. (2006) se reportan resultados de un estudio paleomagnético de magnetismo de rocas y paleointensidades realizado en 24 flujos de lavas que pertenecen al CVMG con edades radiométricas conocidas menores a $3 \mathrm{Ma}$. Entre estos flujos se encuentran volcanes con actividad muy reciente como el volcán Jorullo y el Paricutín. Sus resultados indican que 7 sitios tienen polaridad inversa y 16 sitios tienen polaridad normal. En uno de los sitios se encontró evidencia del registro del evento de polaridad geomagnética normal dentro del Cron Matuyamallamado Olduvai, yen otro sitio se encontró también un posible registro del evento de polaridad Big Lost. Reportan que solo se obtuvieron determinaciones confiables de paleointesnidad en dos de los sitios, el Jorullo (1754-1759) y el Cainjuata (edad $<40 \mathrm{ka}$ ) con valores cercanos al valor actual del CMT.

Gratton et al. (2005) llevaron a cabo un estudio de paleointesidad realizado en lavas históricas delvolcánEl Jorullo, un volcán monogenético formado entre 1759 y 1766 (Tabla 1). Las paleointensidades se obtuvieron mediante el método de microondas de Kono y Ueno (1977) y variaciones del método de Thellier y Thellier (1959). Concluyen que, en términos generales, los valores de la paleointensidad encontrados por ambos métodos $(46.6 \pm 6.3 \mu \mathrm{T})$ corresponde según lo esperado para con valor actual del CMT.

Maciel-Peña et al. (2009) publicaron un estudio paleomagnético realizado en 11 flujos de lava del Volcán Tancítaro, perteneciente al CVMG con edades radiométricas menores a $1 \mathrm{Ma}$. Concluyen que, tanto la posición promedio del polo geomagnético obtenido como su variación paleosecular coinciden con lo esperado durante el Plio-cuaternario para la región de norte América, descartando así la existencia de movimientos tectónicos significativos en al área en el último $1 \mathrm{Ma}$.

Con la finalidad de obtener la paleointensidad Michalk et al. (2010) realizan un estudio en 51 flujos de lava distribuidos a lo largo de la FVTM, la mayoría de ellos con edades menores a $1 \mathrm{Ma}$. De estos sitios, 13 pertenecen al CVMG. Obtienen 32 determinaciones de paleointensidades mediante el método de Multiespecimen, propuesto por Dekkers y Böhnel (2006). Concluyen que sus resultados obtenidos de PI son, en promedio, cerca del $30 \%$ más altos que los valores

Tabla 1. Principales aparatos volcánicos del CVMG, geolocalización y edades radiométricas.

\begin{tabular}{lllcc}
\hline Volcán & Lat. $\left[{ }^{\circ} \mathrm{N}\right]$ & Lon. [ $\left.{ }^{\circ} \mathbf{O}\right]$ & Edad & Error \\
\hline Volcán Paricutín & $19^{\circ} 29^{\prime} 33^{\prime}$ & $102^{\circ} 15^{\prime} 04^{\prime \prime}$ & $1943-1952$ & - \\
Volcán el Jorullo & $18^{\circ} 59^{\prime} 19^{\prime \prime}$ & $101^{\circ} 43^{\prime} 03^{\prime \prime}$ & $1759-1774$ & - \\
Cerro El Jabalí & $19^{\circ} 26^{\prime} 56^{\prime \prime}$ & $102^{\circ} 06^{\prime} 46^{\prime \prime}$ & $3830 \mathrm{AP}$ & 150 años \\
Cerro La Taza & $1^{\circ} 32^{\prime} 20^{\prime \prime}$ & $101^{\circ} 59^{\prime} 33^{\prime \prime}$ & $4700 \mathrm{AP}$ & 200 años \\
Hoya & $19^{\circ} 31^{\prime} 33^{\prime \prime}$ & $101^{\circ} 43^{\prime} 28^{\prime \prime}$ & $8430 \mathrm{AP}$ & 330 años \\
El Huanillo & $19^{\circ} 41^{\prime} 01^{\prime \prime}$ & $101^{\circ} 59^{\prime} 04^{\prime \prime}$ & $9180 \mathrm{AP}$ & 250 años \\
Volcán La Mina & $1^{\circ} 42^{\prime} 45^{\prime \prime}$ & $101^{\circ} 26^{\prime} 02^{\prime \prime}$ & $17170 \mathrm{AP}$ & 430 años \\
El Pueblito & $19^{\circ} 49^{\prime} 29^{\prime \prime}$ & $101^{\circ} 55^{\prime} 24^{\prime \prime}$ & $29000 \mathrm{AP}$ & 3300 años \\
Cerro Las Cabras & $19^{\circ} 49^{\prime} 34^{\prime \prime}$ & $101^{\circ} 53^{\prime} 37^{\prime \prime}$ & $40000 \mathrm{AP}$ & \\
Cerro Pelón & $19^{\circ} 17^{\prime} 52^{\prime \prime}$ & $101^{\circ} 54^{\prime} 47^{\prime \prime}$ & $0.37 \mathrm{Ma}$ & $0.05 \mathrm{Ma}$ \\
Santa Teresa & $20^{\circ} 29^{\prime} 50^{\prime \prime}$ & $100^{\circ} 59^{\prime} 53^{\prime \prime}$ & $2.78 \mathrm{Ma}$ & $0.07 \mathrm{Ma}$ \\
\hline
\end{tabular}

esperados para esta región y edad según recopilaciones y modelos geomagnéticos y concluyen también que, debido a la calidad de sus detreminaciones, este promedio podría tener un significado geomagnético.

Ruiz-Martínez et al. (2010) reportan nuevos datos paloemagnéticos provenientes de 51 sitios distribuidos temporalmente en los últimos $11 \mathrm{Ma}$ y espacialmente a lo largo de la FVTM. Sus resultados son analizados con la finalidad de estudiar la variación paleosecular y establecer posibles implicaciones tectónicas regionales, dividiendo la FVTM en los sectores Central Este y Oeste. 13 de estos sitios se localizan en la parte norte del CVMG y seis en la parte sur, y tres más en los alrededores del lago de Cuitzeo. Concluyen que, en términos generales, los datos paleomagnéticos muestran que la posición del polo paleomagnético en los tres sectores coincide bien con lo esperado según los modelos del CMT y los polos previamente resportados y descartan alguna actividad tectónica regional significativa.

Maciel-Peña et al. (2011) efectuán un estudio paleomagnético realizado en 17 unidades volcánicas con edades radiométricas entre 2.78 a $0.56 \mathrm{Ma}$ localizadas dentro del CVMG. Sus resultados indican que la posición promedio del polo registrado en esas unidades es la misma que la que se espera según los modelos sintéticos globales de deriva polar para el Plio-Cuaternario en la región de Norte América. Reportan también el registro de un posible evento de polaridad normal dentro del Cron Inverso Matuyama, más antiguo que los eventos de Reunion (2.35 Ma) y encuentran también evidencia de la existencia de un campo completamente invertido dentro del Cron Brunhes alrededor de los $0.56 \mathrm{Ma}$.

Michalk et al. (2013) presentaron los resultados de un estudio paleomagnético realizado en 59 flujos de lava distribuidos a lo largo de la FVTM. De los 59 sitios, 21 sitios pertenecen al CVMG con edades de $2.78 \mathrm{Ma}$ a $0.56 \mathrm{Ma}$. Ralizaron también 11 nuevos fechamientos radiométricos. Sus resultados les permiten establecer una correlación con la escala de polaridades geomagnéticas de referencia y aportan evidencia de la posible ocurrencia de cinco excursiones geomagnéticas; tres durante el Cron de Brunhes, un registro de la excursión llamada Big Lost y otra de la excursión Delta/Stage 17.

El trabajo de Maciel-Peña et al. (2014) es un estudio paleomagnético realizado en 37 flujos de lava localizados en la región de Tacámbaro y el Jorullo, estado de Michoacán, en la parte sur del CVGM. Las lavas estudiadas provienen principalmente de volcanes monogenéticos con edades menores a $4 \mathrm{Ma}$. A partir de la posición del polo geomagnético promedio concluyen que no se han presentado movimientos tectónicos significativos en la región desde el emplazamiento de las lavas. Concluyen también que la variación paleosecular observada se ajusta a los modelos geomagnéticos para la misma región y edad. Con las edades asociadas a los flujos de lava establecen una correlación con la escala geomagnética de polaridades destacandose la evidencia de una posible excursión geomagnética Intra Jaramillo.

Con la finalidad de establecer de forma más precisa las edades del emplazamiento de lavas en cuatro unidades volcánicas GarcíaQuintana et al. (2016) realizaron datación paleomagnética a losvolcanes La Taza, El Frijol, Cerro viejo y Chendanas, localizados en los alrededores del lago de Pátzcuaro, Michoacán y con edades radiométricas asociadas menores a 8000 años. A partir de la comparación de las componentes paleomagnéticas de la magnetización (declinación, inclinación e intensidad) presente en las lavas estudiadas con las esperadas según el modelo de campo magnético SHADIF.14k, concluyen que las edades paleomagnéticas que obtienen son cercanas a las esperadas según dataciones radiométricas conocidas para los volcanes La Taza y el Frijol y Chendanas. En el caso de Cerro Viejo se encontró una polaridad magnética inversa, por lo que proponen, esta lava probablemente fue emplazada en tiempo más antiguo a los 14000 años (el alcance de 
modelo SHADIF.14k) y pudiera ser un registro del evento de polaridad geomagnética intermedia denominado Laschamp ya que, aunque no se cuenta con una edad radiométrica para esta erucpión, se conoce por relaciones estratigráficas que su edad debe ser cercano a los $40 \mathrm{ka}$.

García-Ruiz et al. (2017) reportan los resultados de un estudio aeromagnético y paleomagnético realizado en flujos de lava del Volcán Tancítaro, en Michoacán. Se presentan nuevos resultados paleomagnéticos provenientes de ocho flujos de lava, con edades entre los 70 a $950 \mathrm{ka}$, además de incluir los datos previamente reportados por Maciel-Peña et al. (2009) para estudiar con mayor detalle la variación paleosecular para el último $1 \mathrm{Ma}$. Luego de calcular la posición del polo paleomagnético y compararlo con la posición esperada, concluyen que no hay actividad tectónica significativa desde la formación de las lavas y además encuentran nueva evidencia del evento de polaridad magnética Jaramillo.

Mahgoub et al. (2017a) realizan datación paleomagética a cuatro volcanes monogenéticos, La Tinaja, La Palma, Mesa La Muerta y Malpaís, localizados en el área de Tacámbaro-Puruarán, en el sector sureste del CVMG. La datación paleomagnética se realizó a partir de las direcciones paleomagnéticas y las paleointensidades obtenidas arrojando intervalos de edades probables para el emplazamiento de las lavas de La Tinaja entre 3650-3480 a.e.c. (antes de la era común), para la Palma entre 3220-2880 a.e.c. En el caso de Mesa la Muerta encuentran dos intervalos posibles, 2240-2070 a.e.c. y 760-630 a.e.c., y para el caso de Malpaís de Cutzaróndiro el intervalo de edad que proponen es de 420-320 a.e.c.. Las edades encontradas para estos dos últimos tienen relevancia arqueológica ya que corresponden al Pre-Clásico tardío en la escala de tiempo arqueológica mesoamericana por lo que las erupciones pudieron haber tenido algún impacto en las culturas de esa época.

Mahgoub et al. (2017b) presentaron un estudio paleomagnético con fines de datación realizado en cinco flujos de lava del Volcán el Metate el cual se localiza en la parte sur del CVMG. Sus resultados mostraron que las edades de cuatro de los flujos son indistinguibles, sugiriendo que el tiempo de emplazamiento fue muy corto confirmando que se trata de un volcán monogenético con una edad de formación probable entre 1150-1290 e.c. (era común), la cual coincide con la edad radiométrica reportada previamente para el flujo más antiguo.

Mahgoub et al. (2018) realizaron un estudio paleomagnético con fines de datación en cuatro volcanes formados durante el holoceno localizados en el CVMG, El Infiernillo, Malpaís las Víboras, Capaxtiro, y Malpaís Prieto, para establecer con mayor detalle las edades de las erupciones y examinar sus repercusiones arqueológicas y de riesgo volcánico. Solo el volcán El Infernillo tiene una edad radiométrica asociada para la erupción de 1525-1420 a.e.c. Sus resultados indican que la edad para la erupción del volcán El Infernillo es de 1500-1370 a.e.c., la cual coincide con la edad radiométrica y se ubica dentro del periodo Pre- Clásico en la escala de tiempo arqueológica de Mesoamérica. Para el volcán Malpaís Las Víboras reportan un intervalo de edad 1340-940 a.e.c. correspondiente al Pre-Clásico Medio, para el Volcán Capaxtiro la edad más probable es entre $200-80$ a.e.c. y para el volcán Malpaís Prieto entre 830-960 e.c. Señalan que esta erupción pudo ocasionar el abandono de algunos asentamientos de los alrededores, los cuales ya han sido documentados arqueológicamente.

Alva-Valdivia et al. (2019) realizan un estudio y datación paleomagnética en flujos de lava históricos del Volcán El Jorullo. Presentan datos paleomagnéticos direccionales y de paleointensidad obtenidos a partir de muestras de rocas provenientes de cuatro flujos de lava (siete sitios), los cuales fueron formados entre 1759-1766 e.c. Sus resultados indican que tanto la variación secular como la intensidad concuerdan, dentro de sus incertidumbres, con los valores esperados según los modelos globales, por lo que concluyen que el CMT se mantuvo estable en el momento de la erupción.
Mahgoub et al. (2019) analizan mediante un estudio paleomagnético y de paleiontensidades la variación paleosecular del CMT registrada en rocas volcánicas provenientes de 32 volcanes formados en el Cuaternario con edades $<46 \mathrm{ka}, 15$ de los volcanes estudiados pertenecen al CVMG. Obtienen direcciones paleomagnéticas de 32 volcanes y 21 paleontensdides absolutas. Para el análisis de la variación paleosecular incluyen datos previamente publicados seleccionados y son agrupados en intervalos sucesivos de edades entre 2000 e.c. a 1600 a.e.c. y entre 1600-44000 a.e.c. A partir de su análisis concluyen que, para el intervalo más jóven, la variación paleosecular se comporta según lo esperado, mientras que la intensidad del CMT parece mostrar un valor pico de $65 \mu \mathrm{T}$ alrededor de 250 a.e.c. En el caso del intervalo más antiguo, observaron valores pico de intensidad alrededor de 12000 a.e.c., 15000 a.e.c. y 27000 a.e.c., así como valores bajos de intensidad alrededor de 24000-17000 a.e.c. Concluyen también que algunos valores atípicos de inclinación observados entre 26000 a.e.c y 27000 a.e.c podría ser primera evidencia del registro de una excursión geomagnética, ya sea la excursión Mono Lake o bien la excursión recientemente propuesta de Rockall (Channell et al., 2016) pero a falta de mayor control geocronológico no es posible determinar de cual se trata.

El trabajo de Pérez-Rodríguez et al. (2020) trata de establecer con mayor detalle la historia eruptiva del volcán El Metate mediante el método de datación paleomagnética. Se analizaron siete de los 13 flujos de lava asociados al volcán El Metate. Las direcciones paleomagnéticas que obtienen en tres de los cuatro más jóvenes son indistinguibles, mientras que las direcciones observadas en el resto de ellos, a excepción uno, no lograron obtener direcciones paleomagnéticas medias consistentes, lo cual fue atribuido al movimiento de los bloques muestreados después del enfriamiento. El flujo restante arrojó una dirección paleomagnética media ligeramente diferente, estadísticamente distinguible del resto. Seis de las paleointensidades obtenidas muestran valores que concuerdan con lo esperado según los modelos globales de variación del CMT. Con los resultados de la datación, proponen dos etapas de emplazamiento para el volcán El Metate; una primera etapa corta entre 992-1131 e.c., y una segunda etapa más extendida, entre 1431-1600 e.c., lo cual, concluyen, se contrapone con la hipótesis del origen monogenético del volcán El Metate.

\section{ANÁLISIS DE DATOS PALEOMAGNÉTICOS}

Se realizó una revisión exhaustiva de datos paleomagneticos publicados del complejo volcánico de Michoacán-Guanajuato, recopilando direcciones paleomagnéticas de los últimos $5 \mathrm{Ma}$ (Tabla S1 del suplemento electrónico). Inicialmente, se seleccionaron direcciones medias paleomagnéticas con una dispersión moderada de $\alpha_{95} \leq 13^{\circ}$ y con edades radiométricas disponibles. Adicionalmente, se seleccionaron direcciones paleomagnéticas obtenidas con $n \geq 4$ especímenes por sitio (flujo).

Para distinguir entre el régimen geomagnético estable (polaridad normal o invertida) y el campo transicional, se aplicó un ángulo de corte de las paleolatitudes de $45^{\circ}$ y $60^{\circ}$, así como el procedimiento propuesto por Vandamme (1994). Esta discriminación permite realizar un análisis fiel de la variación paleosecular mediante la obtención de la dispersión de polos geomagnéticos virtuales y su comparación con el modelo G de Lawrence et al. (2009), el modelo de Jonhson et al. (2008), así como el modelo TK03 de Tauxe y Kent (2004). Se calculó el valor de la anomalía de la inclinación magnética y se comparó con la curva propuesta por Johnson et al. (2008). Adicionalmente se realizó la prueba de inversión según McFadden y McElhinny (1990), con el fin de establecer si las direcciones normales e inversas son antipodales 
y comparten una dirección común, e incluirlas en el cálculo de los promedios, siempre y cuando la prueba resulte positiva.

El resultado de estos análisis permitió obtener una base de datos conformada por 112 paleodirecciones (Figura 3), con una mayor concentración de datos para el último millón de años (Figura 4). La dirección media obtenida es: Declinación ${ }_{\text {media }}=356.48^{\circ}$, Inclinación $_{\text {media }}$ $=38.84^{\circ}$ y un ángulo de dispersión $\alpha_{95}=8.16^{\circ}$ que corresponde a una posición de polo paleomagnetico virtual de Plat $=87.62^{\circ}$, Plon $=6.77^{\circ}$ y $\alpha_{95}=3.14^{\circ}$. Después de descartar datos transicionales, se obtiene una dirección media $\mathrm{Dm}=356.33^{\circ}$ y $\mathrm{Im}=34.92^{\circ}$ con un $\alpha_{95}=3.66^{\circ}$ $\mathrm{y}$ su polo paleomagnetico Plat $=86.49^{\circ}$, Plon $=3.66^{\circ}, \mathrm{A}_{95}=3.10^{\circ}$. $\mathrm{Al}$ comparar estas posiciones con los polos de referencia para el Cratón de Norteamérica se observa que son indistinguibles (Besse y Courtillot, 2002) y Dm $=1.6^{\circ}, \mathrm{Im}=35.1^{\circ}$, Torsvik et al. (2012) considerando las incertidumbres (Figuras 5 y 6 ).

Las direcciones obtenidas se sometieron a un análisis de dispersión angular para obtener su parámetro $S b$ incluyendo todas las direcciones, y posteriormente removiendo las direcciones correspondientes a eventos de transición con los ángulos de corte de $45^{\circ}, 60^{\circ}$ y el ángulo de corte de Vandamme (1994), utilizando la aproximación de Johnson et al. (2008):

$$
\lambda_{c}=90^{\circ}-\left(1.8 S_{B}+5\right)^{\circ}
$$

El ángulo de corte de Vandamme equivale a $59.77^{\circ}$, que resulta muy similar al ángulo de corte de $60^{\circ}$, por lo que solo se utilizaron los ángulos de corte de $60^{\circ}$ y $45^{\circ}$. Se comparó la dispersión del ángulo de la base de datos con el modelo G de McElhinny y McFadden (1997) y Johnson et al. (2008), así como con el modelo TK03 de Tauxe and Kent (2004). Se observa una dispersión mayor involucrando todos los datos, $y$ al remover las latitudes de polo correspondientes a direcciones transicionales se observa su disminución y su similitud con el Modelo G de Johnson et al. (2008) y McFadden et al. (1991), y al mismo tiempo muy cercanos al TK03 de Tauxe y Kent (2004), (Tabla 2 y Figura 7).

Posteriormente se obtuvo la anomalía de la inclinación con respecto a la esperada por el GAD, obteniendo valores muy cercanos a cero para las tres direcciones medias obtenidas, lo que difiere un poco con respecto a los datos de Johnson et al. (2008) y de la curva propuesta, probablemente debido a la cantidad de datos en las latitudes similares que se tenían al momento de realizar dicho estudio (Figura 8).

Se aplicó la prueba de inversión reportada por McFadden y McElhinny (1990), donde se estimaron los ángulos observados entre las distribuciones normales e invertidas, el ángulo crítico (Tabla 3), los valores de precisión k1, k2 asociados con su distribución, donde fue posible encontrar que las medias direccionales corresponden a una misma distribución. Como es de esperarse, se observa mayor grado de antipodalidad para los datos seleccionados según los ángulos de corte. Una de las características más interesantes del registro del campo geomagnético es la ocurrencia de paleodirecciones intermedias, que contribuyen a la Escala de Tiempo de Inestabilidad Geomagnética (GITS, Singer et al., 2002). Persiste un debate activo entre la comunidad paleomagnética sobre cómo distinguir entre direcciones de variación secular y direcciones de polaridad intermedia (o transicional). Camps et al. (2007) proporcionaron una discusión detallada sobre este tema, mientras que Johnson et al. (2008) adoptaron un ángulo de corte de $45^{\circ}$.

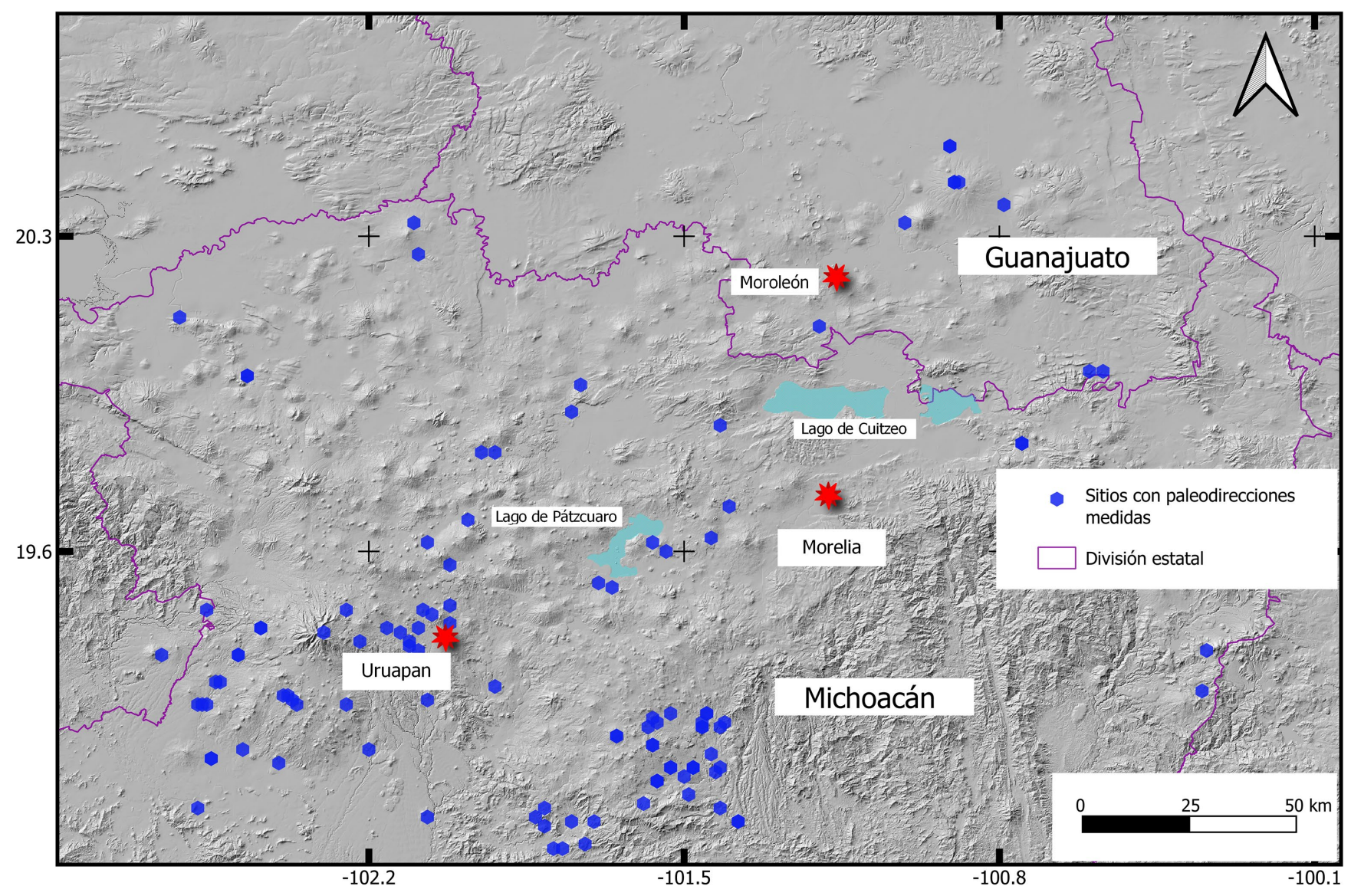

Figura 3. Localización de 112 aparatos volcánicos (principalmente flujos de lava) reportados en la base de datos seleccionados (ver Anexo 1). 

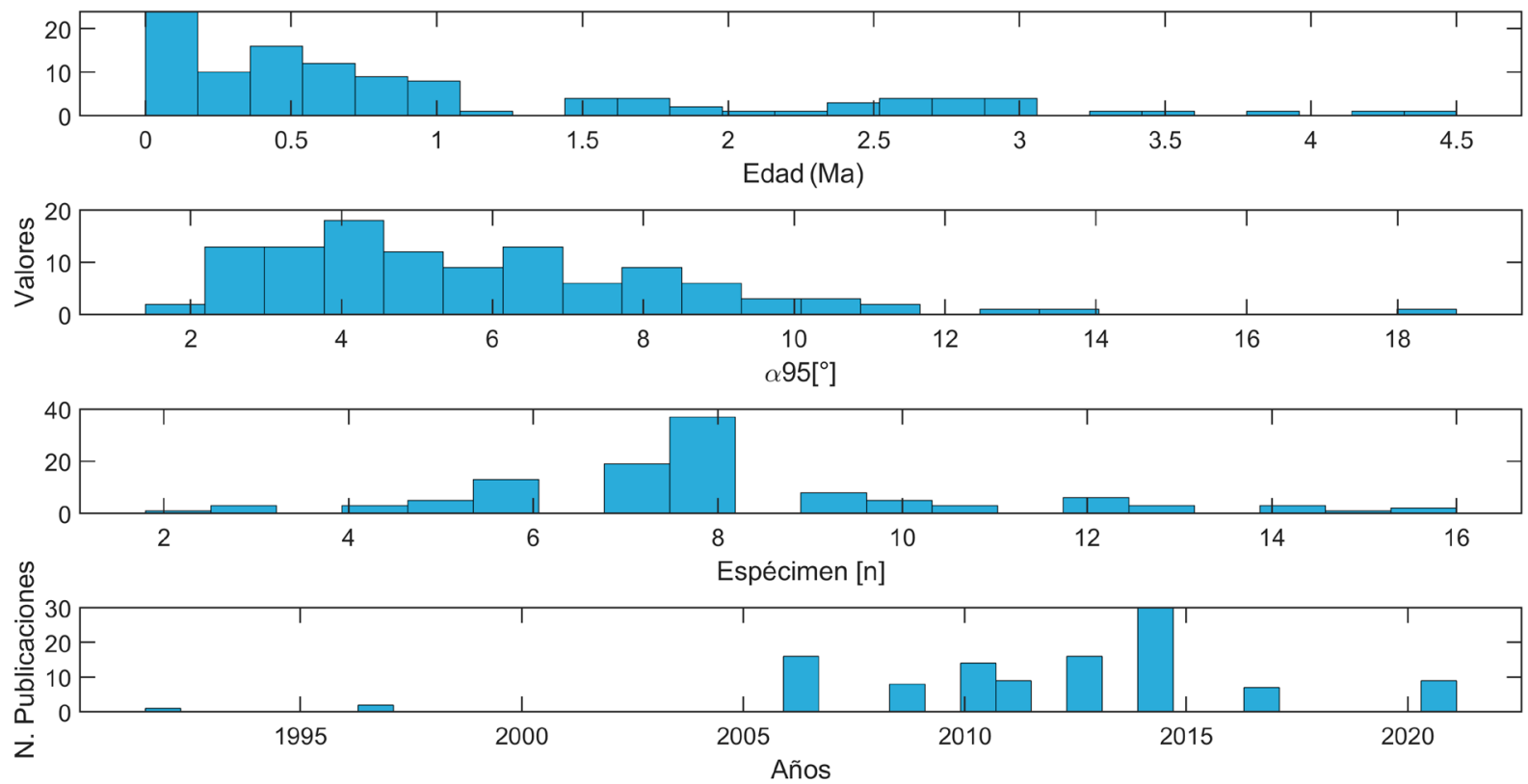

Figura 4. Histogramas de las edades de las direcciones en la base de datos, se indica el valor de a95, el número de especímenes y las publicaciones realizadas hasta la fecha.
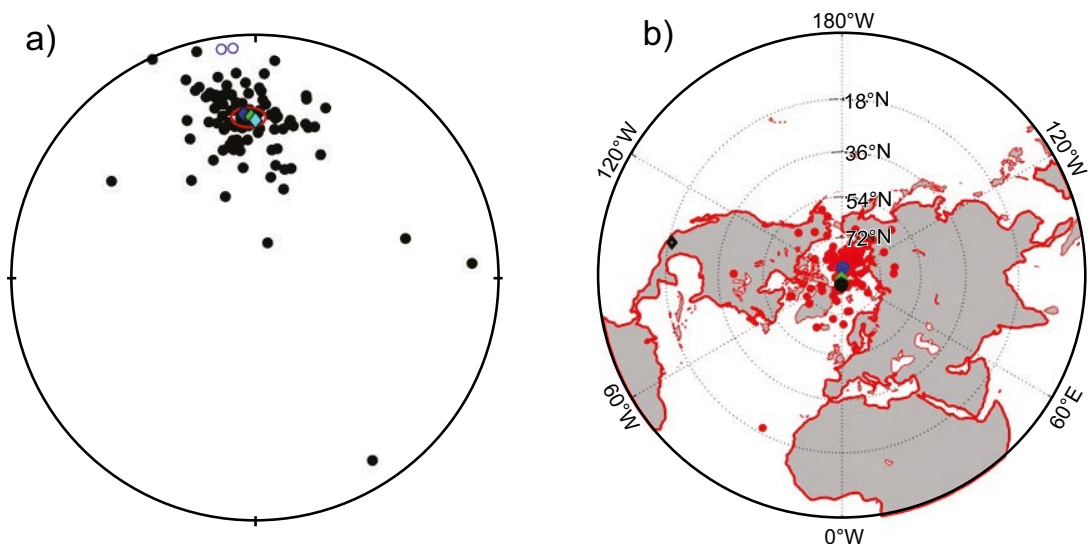

Figura 6. a) Paleodirecion media global obtenida en este estudio, su cono de confianza, comparadas con las direcciones esperadas recalculadas del Cratón de Norteamérica y su dipolo geocéntrico axial (GAD) (azul, verde y magenta), b) Posicion de los polos geomagnéticos virtuales por cada flujo de lava y polo paleomagnético correspondiente (en negro). Comparado con los polos de referencia para el Cratón de Norteamérica (azul y verde).
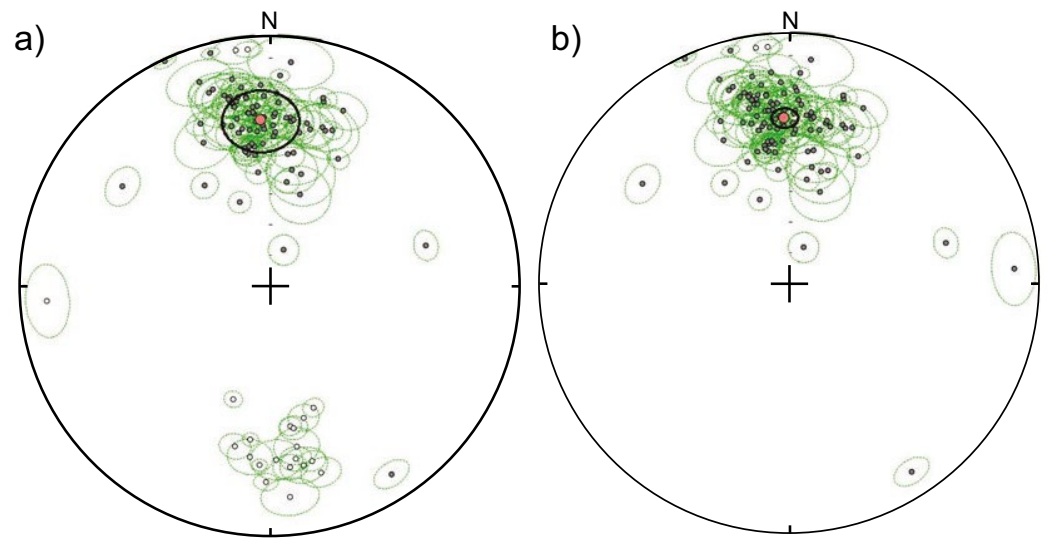

Figura 5. Estereogramas de iguales áreas de paleodireciones medias de flujos de lava asociadas al Campo Volcánico Michoacán-Guanajuato, a) Direcciones normales, transicionales e invertidas, b) direcciones normalizadas. 
Tabla 2. Ángulos de corte de $60^{\circ} \mathrm{y} 45^{\circ}$ (Áng.cort.), de las paleodirecciones medias calculadas (Dm, Im), el número de datos $(\mathrm{N})$, su dispersión (Sbd), así como su dispersión superior (Sbu) e inferior(Sbl), con su cono de confianza $\alpha_{95}$, así como su anomalia de la inclinación $\Delta l$.

\begin{tabular}{lcccccccc}
\hline Áng.cort. & $\mathbf{D m}$ & $\mathbf{I m}$ & $\mathbf{N}$ & Sbd & Sbu & Sbl & $\boldsymbol{\alpha}_{95}$ & $\boldsymbol{\Delta} \boldsymbol{l}$ \\
\hline Todos & $357.67^{\circ}$ & $34.39^{\circ}$ & 106 & 21.44 & 24.87 & 20.79 & $3.53^{\circ}$ & -0.99 \\
$60^{\circ}$ & $357.00^{\circ}$ & $33.20^{\circ}$ & 99 & 13.76 & 16.10 & 13.45 & $2.52^{\circ}$ & -2.18 \\
$45^{\circ}$ & $356.52^{\circ}$ & $33.21^{\circ}$ & 101 & 14.34 & 16.76 & 14.01 & $2.60^{\circ}$ & -2.17 \\
\hline
\end{tabular}

En este trabajo se adoptó este último, que probablemente sea el criterio más estricto. El conocimiento sobre las excursiones geomagnéticas y los eventos de polaridad corta es crucial porque las mayores desviaciones del campo geomagnético de la configuración del dipolo axial se informan en registros sedimentarios, mientras que los registros confiables de rocas volcánicas aún son escasos.

Aunque en la base de datos se reportan registros de varios de flujos de lava con direcciones y polaridades geomagnéticas pertenecientes al régimen geomagnético transicional, solo se discutirá el intervalo comprendido entre las Crones de Bruhnes y Matuyama por su mejor distribución temporal y de mayor calidad en términos paleomagneticos y radiométricos (Figura 9). Por otro lado, aunque existen datos direccionales intermedios de flujos de lava con edades más jóvenes a los 0.03 y $0.025 \mathrm{Ma}$, estos son demasiado jóvenes para ser fechados directamente por el método Ar-Ar, por lo que no es posible evaluar su importancia geomagnética ya que su edad se obtuvo mediante una metodología de datación indirecta basado en isotopos de carbono del material asociado a las erupciones, y no directamente de las lavas.

De 112 unidades de enfriamiento independientes, 4 lavas dieron direcciones con una paleolatitud geomagnética virtual (VGP) $<45^{\circ}$ (direcciones intermedias). Otros dos sitios dentro del cron geomagnético de polaridad normal de Brunhes produjeron direcciones de polaridad inversa. Finalmente, 7 sitios dentro del cron de polaridad inversa de Matuyama arrojaron direcciones cercanas a las actuales.

Tres sitios fechados entre 0.56 y $0.53 \mathrm{Ma}$ (Ban et al., 1992, ConteFasano et al., 2006, Maciel-Peña et al., 2011; Garcia Ruiz et al., 2021) produjeron direcciones de polaridad inversa bien definidas, mientras que un flujo de lava exhibió direcciones de transición. Estas lavas pueden corresponder a cualquiera de las excursiones de West Eifel. De hecho,
Tabla 3. Prueba de inversión involucrando todos los sitios y según los ángulos de corte (Áng.cort.) de $60^{\circ}$ y $45^{\circ}$, los ángulos críticos obtenidos (Áng.crít.), los ángulos observados, los parámetros estadisticos $\mathrm{K} 1$ y K2 de las distribuciones, así como las clases obtenidas por cada distribución.

\begin{tabular}{lcccccc}
\hline Áng.cort. & Áng. crít. & Áng.obs. & Rc & K1 & K2 & Clase \\
\hline Todos & $11.95^{\circ}$ & $4.99^{\circ}$ & 95.66 & 15.74 & 4.25 & Pos. C \\
$60^{\circ}$ & $6.29^{\circ}$ & $1.25^{\circ}$ & 94.21 & 33.72 & 44.22 & Pos. B \\
$45^{\circ}$ & $6.59^{\circ}$ & $0.83^{\circ}$ & 95.87 & 30.20 & 44.22 & Pos. B \\
\hline
\end{tabular}

las direcciones del área volcánica de Eifel se interpretaron inicialmente como un evento geomagnético único (Schnepp y Hradetzky, 1994). Posteriormente, se demostró que el registro contenía hasta cinco eventos entre 730 y $530 \mathrm{ka}$ (Singer et al., 2006). Las excursiones West Eifel 4 y West Eifel 5 fueron fechadas en $555 \pm 4$ ka y $528 \pm 16$ ka, respectivamente (Calvo-Rathert et al., 2013).

Maciel-Peña et al. (2014) reportaron un flujo de lava de polaridad normal con edad de $0.96 \pm 0.07 \mathrm{Ma}$. La misma lava muestreada en una localidad diferente produjo una magnetización de polaridad inversa con un VGPlat igual a $-49^{\circ}$, que está cerca de una dirección de transición (Garcia Ruiz et al., 2017). En cambio, dos flujos estudiados por Michalk et al., (2013) con edades de 0.96 y $1.08 \mathrm{Ma}$, respectivamente, arrojaron direcciones de polaridad normales y, por lo tanto, podrían corresponder al sub-Cron de Jaramillo (Doell y Dalrymple, 1966; Seward, 1974; Laj y Channell, 2007) de 0.99-1.07 Ma. El evento de Jaramillo fue descubierto por Doell y Dalrymple (1966) en rocas volcánicas en la Caldera de los Valles (Santa Fe, Nuevo México); las mismas fueron datadas aproximadamente en $0.9 \mathrm{Ma}$. (Singer et al., 1999). El evento de Jaramillo ha sido ampliamente documentado ya que se ha observado tanto en sedimentos marinos como continentales (Kravchinsky, 2017; Channell et al., 2020), y se considera un evento global (Jacobs, 1994).

Dos flujos de lava estudiados por Maciel-Peña et al. (2014) fechados alrededor de 1.7 Ma y un sitio reportado por Conte-Fasano et al. (2006) con edad 1.8 Ma, podrían corresponder a un sub-Cron Olduvai dentro de cron normal de Matuyama. Aún persiste un debate sobre la edad de la base y la parte superior de Olduvai (Channell et al., 2020). Esto mismo sucede con su duración. Singer et al. (2004) realizaron un estudio en una secuencia de coladas en el Cerro Fraile, en Argentina.

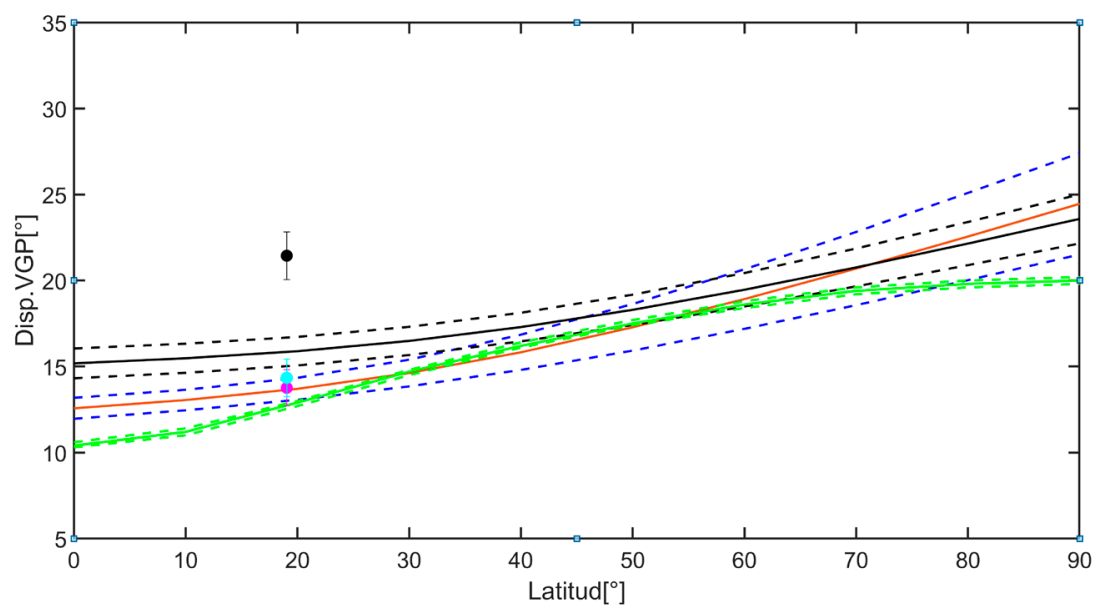

Figura 7. Modelo G de variación paleosecular con la finalidad de comparar la dispersión angular con respecto a la latitud de interés, en negro es el modelo de Johnson et al. (2008), azul McElhinny y McFadden et al. (1997) y en amarillo el TK03 de Tauxe y Kent (2004). La dispersión angular en negro es para todos los sitios sin discriminar, en cian es para el ángulo de corte de $60^{\circ}$ y magenta ángulo de corte de $45^{\circ}$. 


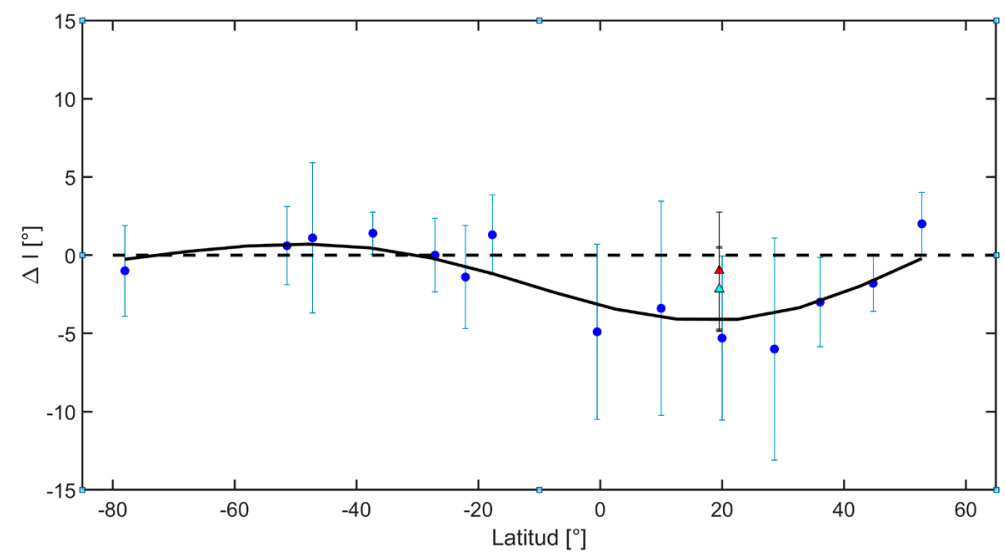

Figura 8. Anomalía de la Inclinación, datos en azul y curva en negro basada en el trabajo de Johnson et al. (2008), con datos en triangulo anomalía de la inclinación para el presente estudio rojo todas, las direcciones, magenta $60^{\circ}$ y cian $45^{\circ}$.

Encontraron flujos con polaridad intermedia fechados en $1.92 \mathrm{Ma}$ superpuestos por un flujo de polaridad normal de 1.85 Ma. Kusu et al. (2016) obtuvieron un registro paleomagnético casi continuo de una inversión de polaridad del Alto Olduvai de un núcleo sedimentario de Yokohama, Japón. La transición de polaridad se determinó entre 1.78-1.77 Ma, observando una secuencia de polaridades N-I-N.

\section{PRINCIPALES CONCLUSIONES Y PERSPECTIVAS}

Una minuciosa revisión de datos paleomagnéticos provenientes de volcanes monogenéticos del CVMG ofrece un registro detallado de la fluctuación de CMT para los últimos $5 \mathrm{Ma}$. Se realizaron análisis paleosecular y campo promediado en el tiempo en 112 unidades de enfriamiento independientes fechadas radiométricamente. Después de separar los flujos de lava con registros de polaridad geomagnética intermedia, la dirección paleomagnética media $\left(\mathrm{Dm}=356.33^{\circ} \mathrm{y}\right.$ Im $=34.92^{\circ}$ con un $\alpha_{95}=3.66^{\circ}$ correspondiente al polo paleomagnetico Plat $=86.49^{\circ}$, Plon $=3.66^{\circ}, \mathrm{A}_{95}=3.10^{\circ}$ ) concuerda bien con las direcciones esperadas para el Plio-Pleistoceno, calculadas a partir de los polos paleomagnéticos de referencia del cratón de América del Norte. Esto descarta cualquier escenario de deformación tectónica o rotación del eje vertical de la Tierra en el intervalo de tiempo involucrado. La dispersión del polo geomagnético virtual coincide, con cierta incertidumbre, con los modelos globales propuestos recientemente para una paleolatitud de $20^{\circ} \mathrm{N}$.

Se adoptó un ángulo de corte de $45^{\circ}$ para la latitud del polo geomagnético virtual con el fin de distinguir los regímenes geomagnéticos estables y de transición. Cuatro sitios de la base de datos actualizada exhibieron direcciones intermedias. Se detectaron flujos de lava con polaridad inversa en dos localidades dentro del crono geomagnético normal de Brunhes, además de siete sitios de polaridad normal en

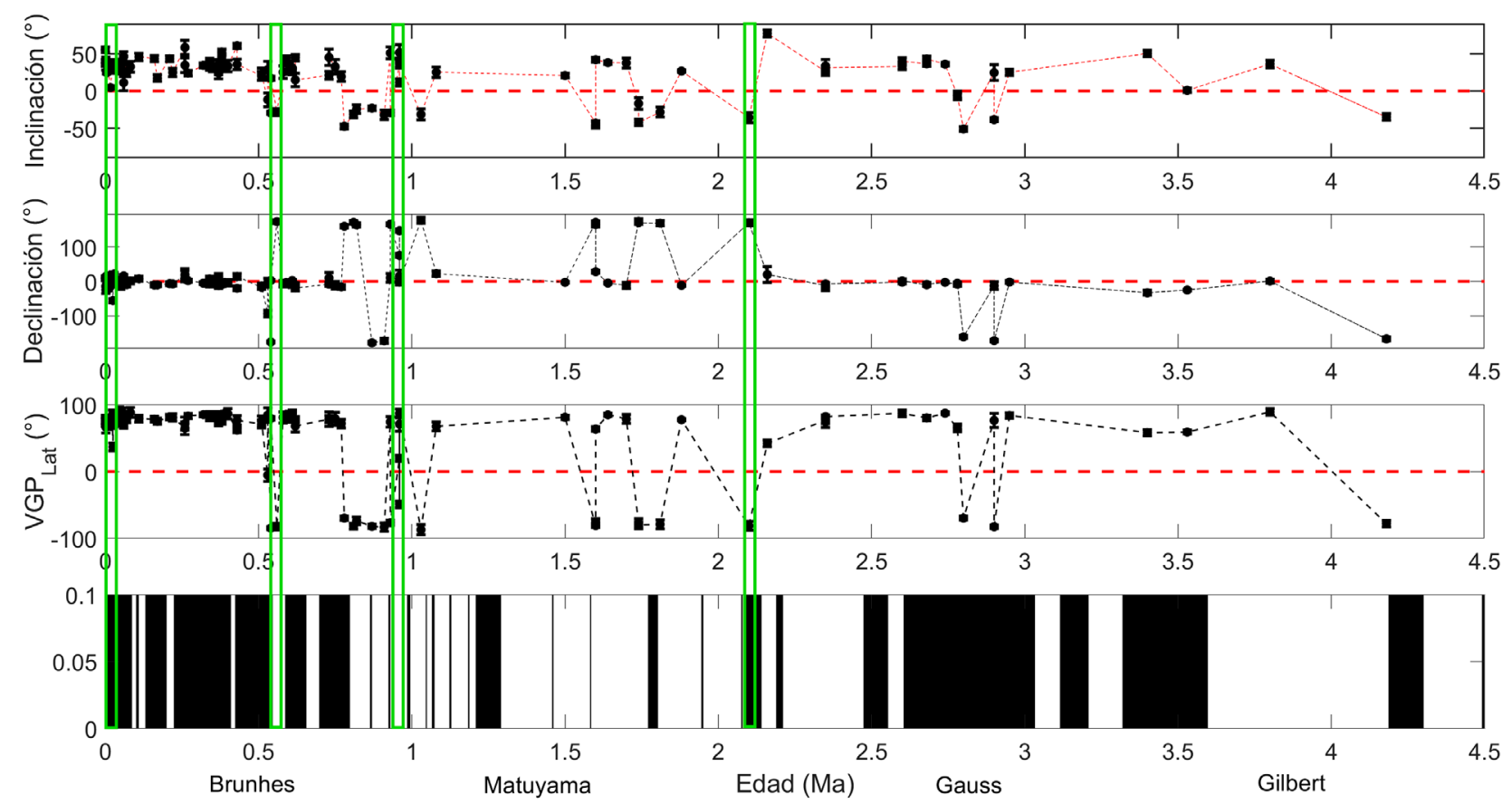

Figura 9. Evolución de la paleolatitud, declinación e inclinación $v s$. edad radiométrica disponible y escala de polaridades paleomagnéticas de referencia. 


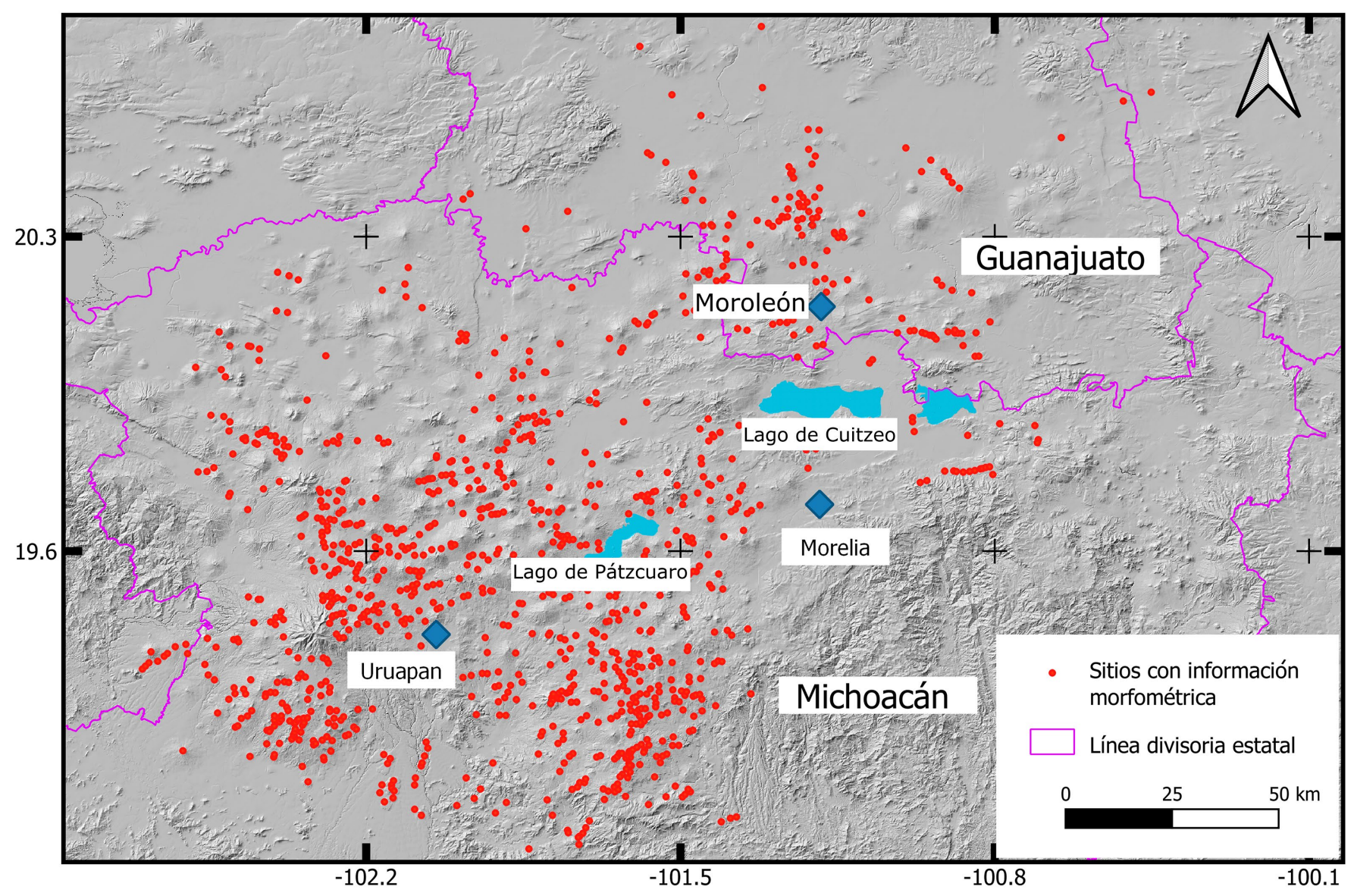

Figura 10. Localización de los 1039 volcanes monogenéticos y sus datos morfométricos recopilados por el Dr. Víctor Hugo Garduño Monroy (inédito).

el cron Matuyama, contribuyendo así a la escala de inestabilidades geomagnéticas.

Es de destacar que la presente base de datos solo representa menos del $10 \%$ de los volcanes monogenéticos, mientras que 1039 aparatos volcánicos dentro del CVMG (Figura 10, Tabla S2 del suplemento electrónico) fueron ubicados por el Dr. Víctor Hugo Garduño durante su fructífera e impresionante vida académica. Este legado, que también incluye valiosos datos morfométricos, podría traducirse en 1039 lecturas instantáneas independientes del campo geomagnético. Por lo tanto, representa una auténtica guía para los estudios paleomagnéticos de las siguientes generaciones.

\section{MATERIAL SUPLEMENTARIO}

Las Tablas S1 y S2 pueden descargarse en el portal web de esta revista $<$ www.rmcg.unam.mx $>$ accediendo a la página de presentación/ resumen de este artículo.

\section{AGRADECIMIENTOS}

Reconocemos todo el trabajo realizado y el apoyo recibido de los revisores anónimos quienes con sus observaciones y sugerencias enriquecieron este trabajo. Esta investigación fue realizada gracias al financiamiento de los proyectos UNAM-PAPIIT-IN101920 y UNAM-PAPIIT-IN113321.

\section{REFERENCIAS}

Alva-Valdivia, L.M., Urrutia-Fucugauchi, J., Böhnel, H., Morán-Zenteno, D.J., 1991, Aeromagnetic anomalies and paleomagnetism in Jalisco and Michoacan, southern Mexico continental margin: Tectonophysics, 192(1-2), 169-190.

Alva-Valdivia, L.M., Rodríguez-Trejo, A., Morales, J., González-Rangel, J.A., Agarwal, A., 2019, Paleomagnetism and age constraints of historical lava flows from the El Jorullo volcano, Michoacán, Mexico: Journal of South American Earth Sciences, 93, 439-448.

Atwater, T., 1970, Implications of plate tectonics for the Cenozoic tectonic evolution of western North America: Geological Society of America Bulletin, 81(12), 3513-3536.

Ban, M., Hasenaka, T., Delgado-Granados, H., Takaoka, N., 1992, K-Ar ages of lavas from shield volcanoes in the Michoacan-Guanajuato volcanic field, Mexico: Geofísica Internacional, 31(4), 467-473.

Besse, J., Courtillot, V., 2002, Apparent and true polar wander and the geometry of the geomagnetic field over the last 200 Myr: Journal of Geophysical Research: Solid Earth, 107(B11), EPM-6.

Böhnel, H., Negendank, J.F.W., 1981, Preliminary results of palaeomagnetic measurements of Tertiary-Quaternary igneous rocks from the eastern part of the Trans Mexican Volcanic Belt: Geofísica Internacional, 20(3), 234-248.

Calvo-Rathert, M., Reyes, B.A., Goguitchaichvili, A., Elguera, J.R., Franco, H., Morales, J., Soto, R., Carrancho, A., Delgado, H., 2013, Rock-magnetic and paleomagnetic results from the Tepic-Zacoalco rift region (western Mexico); Studia Geophysica et Geodaetica, 57(2), 309-331.

Camps, P., Henry, B., Nicolaysen, K., Plenier, G., 2007, Statistical properties of paleomagnetic directions in Kerguelen lava flows: Implications for the late Oligocene paleomagnetic field: Journal of Geophysical Research: 
Solid Earth, 112(B6), DOI:10.1029/2006JB004648

Channell, J.E.T., Harrison, R.J., Lascu, I., McCave, I.N., Hibbert, F.D., Austin, W.E.N., 2016, Magnetic record of deglaciation using FORC-PCA, sortablesilt grain size, and magnetic excursion at $26 \mathrm{ka}$, from the Rockall Trough (NE Atlantic): Geochemestry. Geophysics. Geosystems, 17(5), 1823-1841, doi.org/10.1002/2016GC006300

Channell, J.E., Singer, B.S., Jicha, B.R., 2020, Timing of Quaternary geomagnetic reversals and excursions in volcanic and sedimentary archives: Quaternary Science Reviews, 228, 106114, DOI:10.1016/j.quascirev.2019.106114

Cifuentes, R.M.U., Urrutia-Fucugauchi, J., 1999, Paleomagnetic study of the Valle de Santiago volcanics, Michoacán-Guanajuato volcanic field, Mexico: Geofisica Internacional, 38(4), 217-230.

Clark, K.F., Foster, C.T., Damon, P.E., 1982, Cenozoic mineral deposits and subduction- related magmatic arcs in Mexico: Geological Society of America Bulletin, 93(6), 533-544.

Conte-Fasano, G., Urrutia-Fucugauchi, J., Goguitchaichvili, A., MoralesContreras, J., 2006, Low-latitude paleosecular variation and the timeaveraged field during the late Pliocene and Quaternary-Paleomagnetic study of the Michoacan-Guanajuato volcanic field, Central Mexico: Earth, Planets and Space, 58(10), 1359-1371.

Dekkers, M.J., Böhnel, H.N., 2006, Reliable absolute palaeointensities independent of magnetic domain state: Earth and Planetary Science Letters, 248(1-2), 508-517.

Delgado-Granados, H., Urrutia-Fucugauchi, J., Hasenaka, T., Ban, M., 1995, Southwestward volcanic migration in the western Trans-Mexican Volcanic Belt during the last 2 Ma.: Geofísica Internacional, 34(3), 341-352.

Doell, R.R., Dalrymple, G.B., 1966, Geomagnetic polarity epochs: A new polarity event and the age of the Brunhes-Matuyama boundary: Science, 152(3725), 1060-1061.

Ferrari, L., 2000, Avances en el conocimiento de la Faja Volcánica Transmexicana durante la última década: Boletín de la Sociedad Geológica Mexicana, 53(1), 84-92.

Ferrari, L., Orozco-Esquivel, T., Manea, V., Manea, M., 2012, The dynamic history of the Trans-Mexican Volcanic Belt and the Mexico subduction zone: Tectonophysics, 522, 122-149.

García-Quintana, A., Goguitchaichvili, A., Morales, J., Cervantes-Solano, M., Osorio- Ocampo, S., Macías, J.L., Urrutia-Fucugauchi, J., 2016, Datación magnética de rocas volcánicas formadas durante el Holoceno: caso de flujos de lava alrededor del Lago de Pátzcuaro (campo volcánico Michoacán-Guanajuato): Revista mexicana de ciencias geológicas, 33(2), 209-220.

García-Ruiz, R., Goguitchaichvili, A., Loera, H.L., Cervantes-Solano, M., Urrutia- Fucugauchi, J., Morales-Contreras, J., Maciel-Peña, R., RosasElguera, J., 2017, Paleomagnetism and aeromagnetic survey from Tancitaro volcano (Central Mexico) paleosecular variation at low latitudes during the past 1 Ma: Geofísica internacional, 56(3), 287-304.

García-Ruiz, R., Cervantes-Solano, M., Goguitchaichvili, A., Reyes, D.S., Kravchinsky, V.A., Morales, J., Cejudo., R., Rosas, J., 2021, Semicontinuous paleomagnetic record of the last $1 \mathrm{Ma}$ from radiometrically dated igneous rocks (Trans-Mexican Volcanic Belt and surrounding areas): Journal of South American Earth Sciences, 108, 103195, https://doi.org/10.1016/j. jsames.2021.103195

Goguitchaichvili, A., Conte, G., Urrutia-Fucugauchi, J., Alva-Valdivia, L., Morales, J., González-Morán, T., 2005, Microwave paleointensity analysis of historic lavas from Paricutín volcano, Mexico: Geofísica internacional, 44(3), 231-240.

Gonzalez, S., Sherwood, G., Böhnel, H., Schnepp, E., 1997, Palaeosecular variation in Central Mexico over the last 30000 years: the record from lavas: Geophysical Journal International, 130(1), 201-219.

Gratton, M.N., Goguitchaichvili, A., Conte, G., Shaw, J., Urrutia-Fucugauchi, J., 2005, Microwave palaeointensity study of the Jorullo volcano (Central Mexico). Geophysical Journal International, 161(3), 627-634.

Gubbins, D., 1988, Thermal core-mantle interactions and time-averaged paleomagnetic field: Journal of Geophysical Research, 93(B4), 3413, https://doi.org/10.1029/JB093iB04p03413

Hasenaka, T., Carmichael, I.S., 1985a, The cinder cones of MichoacánGuanajuato, central Mexico: their age, volume and distribution, and magma discharge rate: Journal of Volcanology and Geothermal Research, 25(1-2), 105-124.
Hasenaka, T., Carmichael, I.S., 1985b, A compilation of location, size, and geomorphological parameters of volcanoes of the Michoacan-Guanajuato volcanic field, central Mexico: Geofísica Internacional, 24(4), 577-607.

Hasenaka, T., Carmichael, I.S., 1987, The cinder cones of MichoacanGuanajuato, central Mexico: petrology and chemistry: Journal of Petrology, 28(2), 241-269.

Jacobs, J.A., 1994, Reversals of the Earth's magnetic field: (Vol. 63): Cambridge University Press.

Johnson, C.L., Constable, C.G., Tauxe, L., Barendregt, R., Brown, L.L., Coe, R.S., Layer, P., Mejia, V., Opdyke, N.D., Singer B.S., Staudigel, H., Stone, D.B., 2008, Recent investigations of the $0-5$ Ma geomagnetic field recorded by lava flows: Geochemistry, Geophysics, Geosystems, 9(4), 31pp, https://doi. org/10.1029/2007GC001696

Kennett, J.P., McBirney A.R., Thunell, R.C., 1977, Episodes of Cenozoic volcanism in the Circum-Pacific region: Journal of Volcanology and Geothermal Research, 2, 145-163.

Kono, M., Ueno, N., 1977, Paleointensity determination by a modified Thellier method: Physics of the Earth and Planetary Interiors, 13(4), 305-314.

Kravchinsky, V.A., 2017, Magnetostratigraphy of the Lake Baikal sediments: A unique record of $8.4 \mathrm{Ma}$ of continuous sedimentation in the continental environment: Global and Planetary Change, 152, 209-226.

Kusu, C., Okada, M., Nozaki, A., Majima, R., Wada, H., 2016, A record of the upper Olduvai geomagnetic polarity transition from a sediment core in southern Yokohama City, Pacific side of central Japan: Progress in Earth and Planetary Science, 3(1), 1-13.

Laj, C., Channell, J.E.T., 2007, Geomagnetic excursions, en Kono M. (ed.), Treatise on Geophysics Volume 5, Chapter 10, Geomagnetism: Amsterdam Elsevier, 373-416.

Lawrence, K.P., Constable, C.G., Johnson, C.L., 2006, Paleosecular variation and the average geomagnetic field at $\pm 20^{\circ}$ latitude: Geochemistry, Geophysics, Geosystems, 7(7), 25pp., https://doi.org/10.1029/2005GC001181

Lawrence, K.P., Tauxe, L., Staudigel, H., Constable, C.G., Koppers, A., McIntosh, W., Johnson, C.L., 2009, Paleomagnetic field properties at high southern latitude: Geochemistry, Geophysics, Geosystems, 10(1), https://doi. org/10.1029/2008GC002072

Maciel-Peña, R., Goguitchaichvili, A., Garduño Monroy, V.H., Ruiz Martinez, V.C., Aguilar Reyes, B., Morales, J., Alva-Valdivia, 1, Caballero, M.C., Urrutia-Fucugauchi, J., 2009, Paleomagnetic and rock-magnetic survey of Brunhes lava flows from Tancitaro volcano, México: Geofísica internacional, 48(4), 375-384

Maciel-Peña, R.M., Goguitchaichvili, A., Henry, B., Sánchez-Bettucci, L., Morales, J., Aguilar R.B., Soler-Arechalde, A.M., Calvo-Rathert, M., 2011, Plio-Pleistocene paleomagnetic record from the Michoacan-Guanajuato monogenetic volcanic field (Western México): Studia Geophysica et Geodaetica, 55(2), 311-328.

Maciel-Peña, R., Goguitchaichvili, A., Guilbaud, M.N., Martínez, V.C.R., Rathert, M.C., Siebe, C., Aguilar R.B., Morales, J., 2014, Paleomagnetic secular variation study of Ar-Ar dated lavas flows from Tacambaro Area (Central Mexico): possible evidence of Intra- Jaramillo geomagnetic excursion in volcanic rocks: Physics of the Earth and Planetary Interiors, 229, 98-109.

Mahgoub, A.N., Böhnel, H., Siebe, C., Salinas, S., Guilbaud, M.N., 2017a, Paleomagnetically inferred ages of a cluster of Holocene monogenetic eruptions in the Tacámbaro-Puruarán area (Michoacán, México): implications for volcanic hazards: Journal of Volcanology and Geothermal Research, 347, 360-370.

Mahgoub, A.N., Böhnel, H., Siebe, C., Chevrel, M.O., 2017b, Paleomagnetic study of El Metate shield volcano (Michoacán, México) confirms its monogenetic nature and young age ( $1250 \mathrm{CE})$ : Journal of Volcanology and Geothermal Research, 336, 209-218.

Mahgoub, A.N., Reyes-Guzmán, N., Böhnel, H., Siebe, C., Pereira, G., Dorison, A., 2018, Paleomagnetic constraints on the ages of the Holocene Malpaís de Zacapu lava flow eruptions, Michoacán (México): implications for archeology and volcanic hazards: The Holocene, 28(2), 229-245.

Mahgoub, A.N., Juárez-Arriaga, E., Böhnel, H., Siebe, C., Pavón-Carrasco, F.J., 2019, Late- Quaternary secular variation data from Mexican volcanoes: Earth and Planetary Science Letters, 519, 28-39.

Mammerickx, J., Klitgord, K.D., 1982, Northern East Pacific Rise: Evolution from 25 My BP to the present: Journal of Geophysical Research: Solid 
Earth, 87(B8), 6751-6759.

McElhinny, M.W., McFadden, P.L., 1997, Palaeosecular variation over the past $5 \mathrm{Myr}$ based on a new generalized database: Geophysical Journal International, 131(2), 240-252.

McFadden, P.L., McElhinny, M.W., 1990, Classification of the reversal test in palaeomagnetism: Geophysical Journal International, 103(3), 725-729.

McFadden, P.L., Merrill, R.T., McElhinny, M.W., Lee, S., 1991, Reversals of the Earth's magnetic field and temporal variations of the dynamo families: Journal of Geophysical Research: Solid Earth, 96(B3), 3923-3933.

Michalk, D.M., Biggin, A.J., Knudsen, M.F., Böhnel, H.N., Nowaczyk, N.R., Ownby, S., López-Martínez, M., 2010, Application of the multispecimen palaeointensity method to Pleistocene lava flows from the Trans-Mexican Volcanic Belt: Physics of the Earth and Planetary Interiors, 179(3-4), 139-156.

Michalk, D.M., Böhnel, H.N., Nowaczyk, N.R., Aguírre-Diaz, G.J., LópezMartínez, M., Ownby, S., Negendank, J.F., 2013, evidence for geomagnetic excursions recorded in Brunhes and Matuyama Chron lavas from the trans-Mexican volcanic belt: Journal of Geophysical Research: Solid Earth, 118(6), 2648-2669.

Morales, J., Goguitchaichvili, A., Valdivia, L. A., Gratton, M.N., Fucugauchi, J.U., Rosas- Elguera, J., Soler, A.M., 2003, An attempt to determine the microwave paleointensity on historic Paricutín volcano lava flows, Central México: Geofísica Internacional, 42(1), 95-100.

Nixon, G.T., Demant, A., Armstrong, R.L., Harakal, J.F., 1987, K-Ar and geologic data bearing on the age and evolution of the Trans-Mexican Volcanic Belt: Geofísica Internacional, 26, 109-158.

Osorio-Ocampo, S., Macías, J.L., Pola, A., Cardona-Melchor, S., Sosa-Ceballos, G., Garduño-Monroy, V.H., Layer, P., García-Sánchez, L., Perton, M., Benowitz, J., 2018, The eruptive history of the Pátzcuaro Lake area in the Michoacán Guanajuato Volcanic Field, central México: Field mapping, $\mathrm{C}-14$ and ${ }^{40} \mathrm{Ar} /{ }^{39} \mathrm{Argeoch}$ ronology: Journal of Volcanology and Geothermal Research, 358, 307-328.

Pérez-Rodríguez, N., Morales, J., Guilbaud, M.N., Goguitchaichvili, A., CejudoRuiz, R., Hernández-Bernal, M.S., 2020, Reassessment of the eruptive chronology of El Metate shield volcano (central-western Mexico) based on a comprehensive rock-magnetic, paleomagnetic and multi-approach paleointensity survey: Quaternary Geochronology, 55, 101031, https:// doi.org/10.1016/j.quageo.2019.101031

Rosas-Elguera, J., Urrutia-Fucugauchi, J., 1992, Magnetoestratigrafía volcánica de la zona geotérmica Ixtlán de los Hervores-Los Negritos, Michoacán, México: Resultados preliminares: Geofísica Internacional, 31(4), 431-442.

Rosas-Elguera, J., Alva-Valdivia, L.M., Goguitchaichvili, A., Urrutia-Fucugauchi, J., Ortega-Rivera, M.A., Prieto, J.C.S., Lee, J.K., 2003, Counterclockwise rotation of the Michoacan Block: implications for the tectonics of western México: International Geology Review, 45(9), 814-826.

Ruiz-Martínez, V.C., Urrutia-Fucugauchi, J., Osete, M.L., 2010, Palaeomagnetism of the Western and Central sectors of the Trans-Mexican volcanic belt implications for tectonic rotations and palaeosecular variation in the past 11 Ma: Geophysical Journal International, 180(2), 577-595.

Seward, D., 1974, Age of New Zealand Pleistocene substages by fission-track dating of glass shards from tephra horizons. Earth and planetary science letters, 24(2), 242-248.
Schnepp, E., Hradetzky, H., 1994, Combined paleointensity and 40Ar/39Ar age spectrum data from volcanic rocks of the West Eifel field (Germany): evidence for an early Brunhes geomagnetic excursion: Journal of Geophysical Research: Solid Earth, 99(B5), 9061-9076.

Shaw, J., 1974, A new method of determining the magnitude of the paleomagnetic field: Application to five historic lavas and five archaeological samples: Geophysical Journal International, 39(1), 133-141, https://doi. org/10.1111/j.1365-246X.1974.tb05443.x

Singer, B.S., Hoffman, K.A., Chauvin, A., Coe, R.S., Pringle, M.S., 1999, Dating transitionally magnetized lavas of the late Matuyama Chron: Toward a new 40Ar/39Ar timescale of reversals and events: Journal of Geophysical Research: Solid Earth, 104(B1), 679-693.

Singer, B.S., Relle, M.K., Hoffman, K.A., Battle, A., Laj, C., Guillou, H., Carracedo, J.C., 2002, Ar/Ar ages from transitionally magnetized lavas on La Palma, Canary Islands, and the geomagnetic instability timescale. Journal of Geophysical Research: Solid Earth, 107(B11), EPM-7, doi:10.1029/2001JB001613

Singer, B.S., Ackert Jr, R.P., Guillou, H., 2004, ${ }^{40} \mathrm{Ar} /{ }^{39} \mathrm{Ar}$ and $\mathrm{K}-\mathrm{Ar}$ chronology of Pleistocene glaciations in Patagonia: Geological Society of America Bulletin, 116(3-4), 434-450.

Singer, B. S., Guillou, H., Zhang, X., Schnepp, E., Hoffman, K. A., 2006, Multiple Brunhes Chron Excursions Recorded in the Eifel Volcanic Field: AGU Fall Meeting Abstracts abstract ID GP21A-1301.

Tauxe, L., Kent, D.V., 2004, A simplified statistical model for the geomagnetic field and the detection of shallow bias in paleomagnetic inclinations: Was the ancient magnetic field dipolar?: Washington DC, American Geophysical Union Geophysical Monograph Series, 145, 101-115.

Thellier, E., Thellier O., 1959, Sur l'intensite du champ magnetique terrestre dans le passé, historique et geologique: Annales de Geophysique, 15, 285-376.

Torsvik, T.H., Van der Voo, R., Preeden, U., Mac Niocaill, C., Steinberger, B., Doubrovine, P.V., Douwe, J.J., Mathew, D., Gaina, C., Thoveri, E., Meert P.G., McCausland, J.A.P., Cocks, L.R.M., 2012, Phanerozoic polar wander, palaeogeography and dynamics: Earth- Science Reviews, 114(3-4), 325-368.

Urrutia-Fucugauchi, J., Alva-Valdivia, L.M., Goguitchaichvili, A., Rivas, M.L., Morales, J., 2004, Palaeomagnetic, rock-magnetic and microscopy studies of historic lava flows from the Paricutin volcano, Mexico: implications for the deflection of palaeomagnetic directions: Geophysical Journal International, 156(3), 431-442.

Vandamme, D., 1994, A new method to determine paleosecular variation: Physics of the Earth and Planetary Interiors, 85(1-2), 131-142.

Manuscrito recibido: marzo 1, 2021

Manuscrito corregido recibido: septiembre 14, 2021

Manuscrito aceptado: octubre 6, 2021 